\title{
The Lateral Crural Insertion Graft for External Nasal Valve Collapse
}

\author{
Dominic Vernon, MD \\ Assistant Professor, Facial Plastic and Reconstructive Surgery \\ Division of Otolaryngology - Head and Neck Surgery \\ Indiana University School of Medicine \\ 1130 W Michigan Street, Fesler Hall, Suite 400 \\ Indianapolis, IN, 46202 \\ dovernon13@gmail.com \\ Andrew Lee, MD \\ Division of Otolaryngology - Head and Neck Surgery \\ Johns Hopkins School of Medicine \\ $601 \mathrm{~N}$ Caroline St $6^{\text {th }}$ Floor \\ Baltimore, MD 21287 \\ alee167@jhmi.edu \\ Ira Papel, MD \\ Professor, Facial Plastic and Reconstructive Surgery \\ Division of Otolaryngology - Head and Neck Surgery \\ Johns Hopkins School of Medicine \\ 1838 Greene Tree Road, Suite 370 \\ Baltimore, MD 21208 \\ idpmd@aol.com
}

Word Count: 767

This is the author's manuscript of the article published in final edited form as:

Vernon, D., Lee, A., \& Papel, I. (2020). Lateral Crural Insertion Graft for External Nasal Valve Collapse. Facial Plastic Surgery \& Aesthetic Medicine, 22(2), 110-111. https://doi.org/10.1089/fpsam.2019.29010.ver 


\section{Keywords}

- External nasal valve

- Nasal valve repair

- Nasal obstruction

\section{Introduction}

Nasal valve collapse is an important contributor to nasal airway obstruction and can be divided into internal and external components. It is most helpful to think of the external valve in terms of its anatomical borders, with the caudal septum and medial crura medially, nasal sill caudally, and alar rim/alar fibrofatty tissue anterolaterally..$^{1,2,3,5}$

The choice of graft for support of the external nasal valve is dependent on a thorough examination of the external nasal valve and lower lateral cartilages. Patients with only mild to moderate dynamic collapse may require only a rim or batten graft for additional support. Those with severely malpositioned cartilages may be at the other end of the spectrum, requiring complete disarticulation and repositioning of the lower lateral cartilages with lateral crural struts. ${ }^{1,6}$ In the senior author's practice, we have noted many patients with severe sidewall collapse requiring strong lateral support but without a need for complete disarticulation of the lower lateral cartilage. The senior author's preferred technique in this patient population is a modification of the lateral crural strut graft, which we term the lateral crural insertion graft. With this modification, strong support of the lateral wall is achieved while minimizing unnecessary dissection. The technique and placement is also more efficient, requiring less operating time than the traditional strut graft.

\section{Surgical Technique}

A thorough examination of the patient is crucial for proper graft selection. All components contributing to nasal airway obstruction must be evaluated, including septal deviation, turbinate hypertrophy, internal valve collapse, and external valve collapse. Patients undergoing the lateral crural insertion graft 
ideally have dynamic external nasal valve collapse. Concurrent septal deviation and turbinate hypertrophy should be addressed at the time of external nasal valve repair.

An open approach is used for the placement of the graft in all patients, utilizing standard transcolumellar and marginal incisions. Septal or auricular cartilage is harvested for grafting material While some reports describe auricular cartilage as too weak for placement of lateral grafts, the senior author finds its strength to be more than adequate for this modified technique. ${ }^{1,6}$

Similar to the standard lateral crural strut, soft tissue pockets are created along the alar groove or just caudal to this area for placement of the lateral end of the grafts. Unlike the traditional strut graft, the lower lateral cartilage is not dissected completely free from its attachment to the vestibular skin. Rather, a vertical incision is made in the lateral aspect of the lower lateral cartilage, leaving behind 1-2 millimeters attached to the underlying skin (Figure 1A, Video1). A plane is then easily developed with scissor dissection between the vestibular skin and the lower lateral cartilage and carried from lateral to medial. The caudal and cephalad portions of the lower lateral cartilage are not separated from the vestibular skin. Rather, a precise pocket is created for insertion of the graft, preserving as much of the attachment of the lower lateral cartilage to the underlying skin as possible (Figure 1B, Video 1).

The medial end of the septal or auricular cartilage graft is inserted in this pocket, deep to the lower lateral cartilage but superficial to the vestibular skin. The amount of overlap between the cartilages can be adjusted based on the amount of support required (Figure 1C, Figure 2). Mattress sutures are placed through vestibular skin, the graft, and the lower lateral cartilage to secure the medial end of the graft in place (Video 1). These sutures also obviate the need for sidewall splints, as conservative dissection of vestibular skin has been performed. The lateral end of the graft is placed in the previously created soft tissue pocket, similar to placement with the standard lateral crural strut graft (Figure 1D, Video 1). If caudal rotation of the crural complex is necessary, this is accomplished with placement of the graft into 
these caudal pockets, similar to an alar strut graft. Any recurvature of the lower lateral cartilage into the nasal airway is also easily addressed. The insertion graft is placed deep to the crura, which supports and flattens the lower lateral cartilage.

Patients follow up one week postoperatively for columella suture removal and at one and three month intervals postoperatively to assess for improvement in nasal breathing.

\section{Conclusion}

The lateral crural insertion graft is a modification of the lateral crural strut graft that limits the amount of vestibular skin and lateral crural dissection. This method provides the strength of a lateral crural strut graft but is technically easier and minimizes postoperative edema and surgical time. It is ideal for patients with moderate to severe external valve collapse and weak or concave lower lateral cartilage requiring underlying support.

\section{Acknowledgements:}

Dominic Vernon, MD: Manuscript drafting and revisions

Andrew Lee, MD: $\quad$ Video file and schematic creation and editing

Ira Papel, MD: $\quad$ Originator of surgical technique, manuscript revisions

\section{References}

1. Samra S, Steitz JT, Hajnas N, Toriumi DM. Surgical Management of Nasal Valve Collapse. Otolaryngol Clin N Am. 2018;51:929-944.

2. Hamilton GS. The External Nasal Valve. Facial Plast Surg Clin N Am. 2017;25:179-194.

3. Motamedi KK, Stephan, SJ, Ries WR. Innovations in Nasal Valve Surgery. Curr Opin Otolaryngol Head Neck Surg. 2016;24:31-36.

4. Most SP. Comparing Methods for Repair of the External Valve. JAMA Facial Plast Surg. 2015;17:345-346. 
5. Speilmann PM, White PS, Hussain SS. Surgical Techniques for the Treatment of Nasal Valve Collapse: A Systemic Review. Laryngoscope. 2009;119:1281-1290

6. Toriumi DM, Asher SA. Lateral Crural Repositioning for Treatment of Cephalic Malposition. Facial Plast Surg Clin N Am. 2015;23:55-71.

7. Toriumi DM, Josen J, Weinberger M, Tardy ME. Use of Alar Batten Graft for Correction of Nasal Valve Collapse. Arch Otolaryngol Head Neck Surg. 1997;123:802-808.

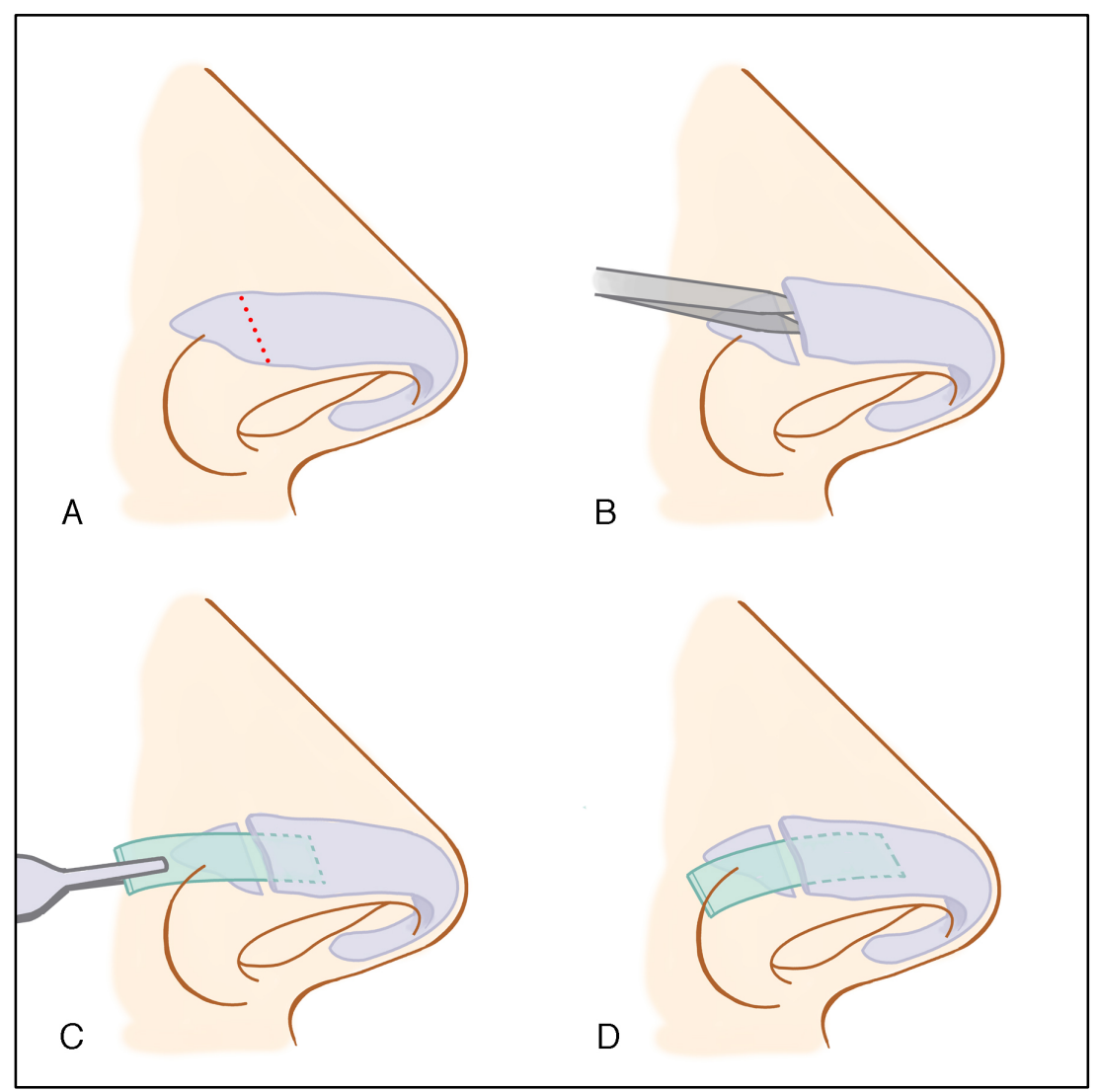

Figure 1. Schematic of surgical steps for insertion graft. 


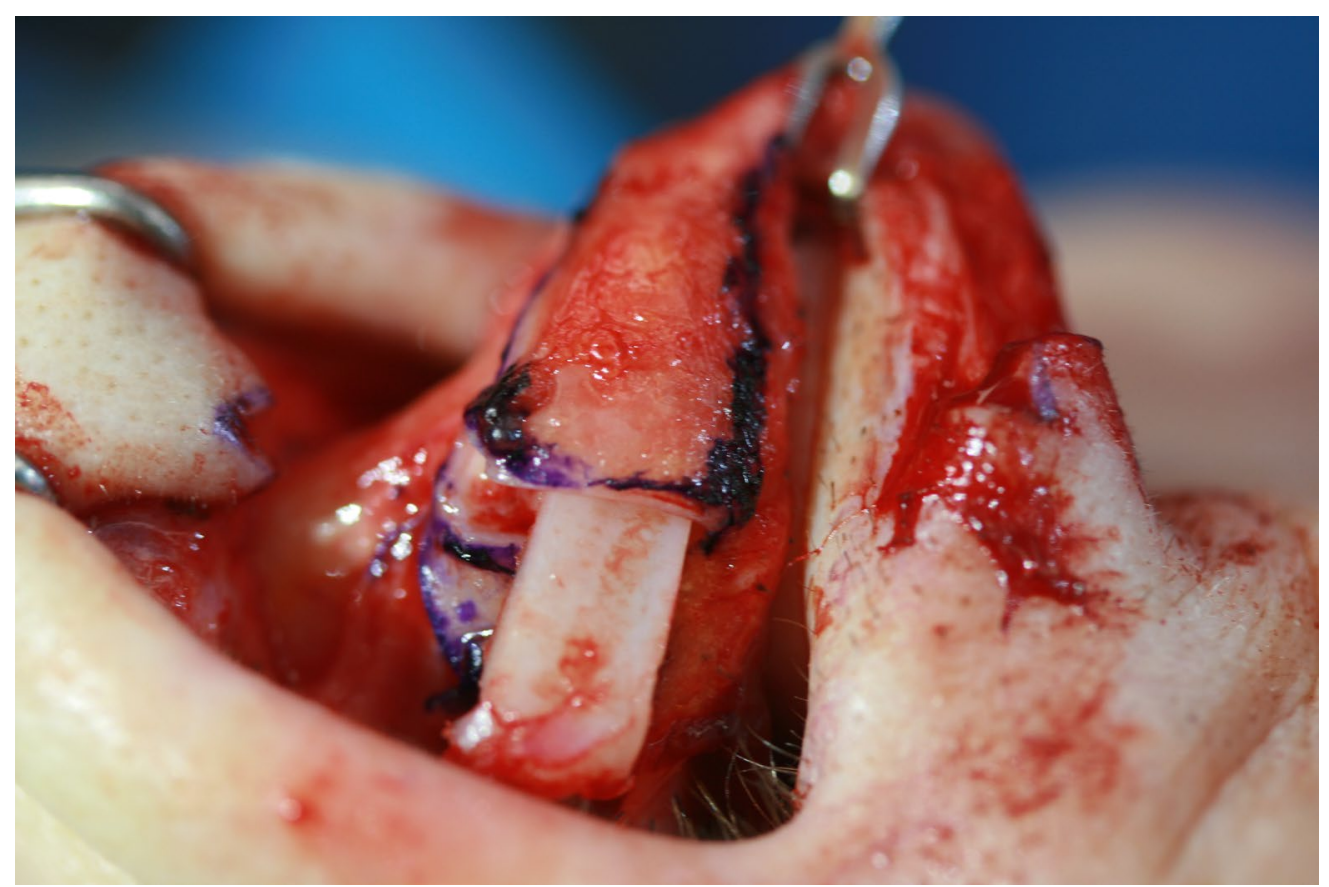

Figure 2. Placement of insertion graft deep to lower lateral cartilage in precise pocket.

Video 1. Lateral Crural Insertion Graft Technique

Narrated video demonstrating the steps for placement of the lateral crural insertion graft. .$m p 4$ format 